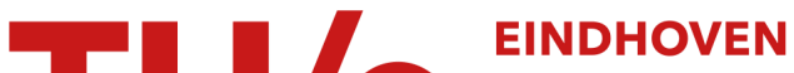 UNIVERSITY OF TECHNOLOGY
}

\section{A BGO detector for catalysis studies with positron emitters}

\section{Citation for published version (APA):}

Mangnus, A. V. G., IJzendoorn, van, L. J., de Goeij, J. J. M., \& Voigt, de, M. J. A. (1995). A BGO detector for catalysis studies with positron emitters. In R. C. Trendler (Ed.), NSS : IEEE nuclear science symposium and medical imaging : conference records, 1995, Norfolk, Va., October 30 to November 5 (pp. 333-336). Institute of Electrical and Electronics Engineers. https://doi.org/10.1109/NSSMIC.1994.474403

DOI:

10.1109/NSSMIC. 1994.474403

Document status and date:

Published: 01/01/1995

\section{Document Version:}

Publisher's PDF, also known as Version of Record (includes final page, issue and volume numbers)

\section{Please check the document version of this publication:}

- A submitted manuscript is the version of the article upon submission and before peer-review. There can be important differences between the submitted version and the official published version of record. People interested in the research are advised to contact the author for the final version of the publication, or visit the $\mathrm{DOI}$ to the publisher's website.

- The final author version and the galley proof are versions of the publication after peer review.

- The final published version features the final layout of the paper including the volume, issue and page numbers.

Link to publication

\section{General rights}

Copyright and moral rights for the publications made accessible in the public portal are retained by the authors and/or other copyright owners and it is a condition of accessing publications that users recognise and abide by the legal requirements associated with these rights.

- Users may download and print one copy of any publication from the public portal for the purpose of private study or research.

- You may not further distribute the material or use it for any profit-making activity or commercial gain

- You may freely distribute the URL identifying the publication in the public portal.

If the publication is distributed under the terms of Article 25fa of the Dutch Copyright Act, indicated by the "Taverne" license above, please follow below link for the End User Agreement:

www.tue.nl/taverne

Take down policy

If you believe that this document breaches copyright please contact us at:

openaccess@tue.nl

providing details and we will investigate your claim. 


\title{
A BGO Detector for Catalysis Studies with Positron Emitters
}

\author{
A.V.G. Mangnus ${ }^{1}$, L.J. van IJzendoorn ${ }^{1}$, J.J.M. de Goeij ${ }^{2}$ and M.J.A. de Voigt ${ }^{1}$ \\ ${ }^{1}$ Eindhoven University of Technology, P.O. Box 513, 5600 MB Eindhoven, The Netherlands \\ ${ }^{2}$ Interfacultary Reactor Institute, Delft University of Technology, Mekelweg 15, 2629 JB Delft, \\ The Netherlands
}

\begin{abstract}
A detector for measurement of the activity distribution of positron emitters in a catalyst reactor has been designed and built. The detector consists of two banks of each ten BGO scintillation crystals. The activity distribution is measured as a function of time and position along the reactor. The attainable position resolution along the reactor is $3 \mathrm{~mm}$ and the minimum sampling time for the measurement of an activity profile is 1 second. The detector has a high sensitivity and the flexibility to be used with catalyst reactors of different types and sizes.
\end{abstract}

\section{INTRODUCTION}

In catalysis, reaction kinetics are often studied with transient techniques, in which a pulse of molecules labeled with (radio)isotopes is injected into a catalyst reactor operating under steady state conditions. From the composition of the reaction products as a function of time information about reaction mechanisms and rates can be obtained. For several reasons the use of positron emitting isotopes in kinetic studies with catalysts is attractive: the positron emitters ${ }^{11} \mathrm{C}\left(\mathrm{t}_{1 / 2}=20.4\right.$ $\min ),{ }^{13} \mathrm{~N}\left(\mathrm{t}_{1 / 2}=10.0 \mathrm{~min}\right)$ and ${ }^{15} \mathrm{O}\left(\mathrm{t}_{1 / 2}=2.0 \mathrm{~min}\right)$ offer the possibility of labeling many chemically interesting molecules, while the short half-lives of these positron emitters give high specific activities, which implies that only very small amounts of labeled reactants have to be synthesized and injected. Furthermore the energy of the annihilation photons is high enough to penetrate the reactor wall and an eventual oven placed around the reactor, which allows in-situ measurements of residence times of reaction intermediates on the catalyst. Activity profiles in combination with reaction modelling can be used to determine the separate reaction steps and rates.

It has been proved $[1,2]$ that positron emitters can indeed be used to study reaction kinetics in catalysts. Moreover, the experiments showed that it was possible to model the reaction kinetics measured under normal reactor operation conditions with reaction parameters obtained with surface science techniques under laboratory conditions [2]. These experiments were carried out with a commercial PET-camera [1], which was designed for patient care and had a three-dimensional imaging capability. As the concentration gradients in a catalyst reactor in radial direction can mostly be neglected compared to those in axial direction, one-dimensional imaging (profiling) will suffice in most cases, which eliminates the need for a complex and expensive PET-camera. In this paper a new detector will be described, which has been designed to measure the activity distribution of positron emitters over a catalyst reactor as a function of position (in one dimension) and time. The choice for a detector especially designed for one-dimensional imaging (PEP-detector, Positron Emission Profiling) offers the possibility to obtain a position resolution in axial direction of the order of a few $\mathrm{mm}$ with a high sensitivity and which is suited to catalyst reactors of different types and sizes.

\section{DETECTOR REQUIREMENTS}

The detector configuration is determined by the requirements for profiling the residence time distribution of an activity pulse of positron emitters over different catalyst reactors. Lenghts of the catalyst beds to be studied will vary from a few $\mathrm{cm}$ to about $50 \mathrm{~cm}$, dependent on the type of reaction and flow conditions. The diameter of the reactor system is mainly determined by the oven placed around the catalyst tube to keep the catalyst at a fixed temperature. The activitity distribution will have to be measured as a function of position and time by means of coincident detection of the two photons emitted in opposite directions upon annihilation of a positron. The positron range imposes a fundamental limit to the position resolution that can be reached (and thus on the minimum length of the catalyst bed that can be profiled, which must be a few $\mathrm{cm}$ 's). The maximum range of the emitted positron before annihilation depends on the catalyst matter; for a typical catalyst support (silica) the maximum ranges of positrons emitted by ${ }^{11} \mathrm{C},{ }^{13} \mathrm{~N}$ and ${ }^{15} \mathrm{O}$ are respectively $1.8,2.3$ and $3.5 \mathrm{~mm}$.

The maximum activity of the pulse injected into the reactor is limited by the radiochemical yield and the timescale required for the labeling of the reactant molecules and by the maximum injection volume that can be used without disturbing the steady state of the reactor. Furthermore both the catalyst tube (often with a reactor wall made of stainless steel to withstand the high pressures needed) and the oven (mostly an aluminum heating block) will cause absorption and Compton scattering of the photons. Therefore the sensitivity of the detector for annihilation photons must be high and an energy selection of the photons is desirable, since scattering prevents a proper reconstruction of the annihilation position. 
The required sampling time of the detector is determined by the time required to reconstruct an activity profile with enough statistics and by the timescale on which the reaction to be studied will take place. A mininum sampling time of one second is considered to be sufficient to fullfil both demands.

\section{DETECTOR CONFIGURATION}

Although multiwire proportional chambers have been used for nonmedical applications of positron emitters [3], they were considered to be unsuited for this detector because of their relatively low sensitivity for annihilation photons and their restricted energy selection capabilities. The highest detection sensitivity is reached with inorganic scintillators but the relatively poor energy resolution hampers the energy selection of the detected photons and thus possibly the position resolution. To obtain a position resolution of a few $\mathrm{mm}$ with scintillators, the detector consists of small, rectangular scintillation crystals individually coupled to a photomultiplier. Coincident annihilation photons are detected by placing crystals opposite to each other. To keep the number of crystals as low as possible and for ease of construction the crystals are grouped in two separate banks. To evaluate the performance for sensitivity and position resolution of different scintillators Monte-Carlo simulations with the EGS4-code [4] were carried out. The geometry used in these simulations is shown in Fig. 1 ; it consists of a stainless steel reactor which is filled with some catalyst matter and which is surrounded by an aluminum oven. The outside of the oven is heat-isolated (with glass wool) and cooled (with water) to prevent the scintillation crystals from heating up and thus allowing a close distance between the reactor and the detector.

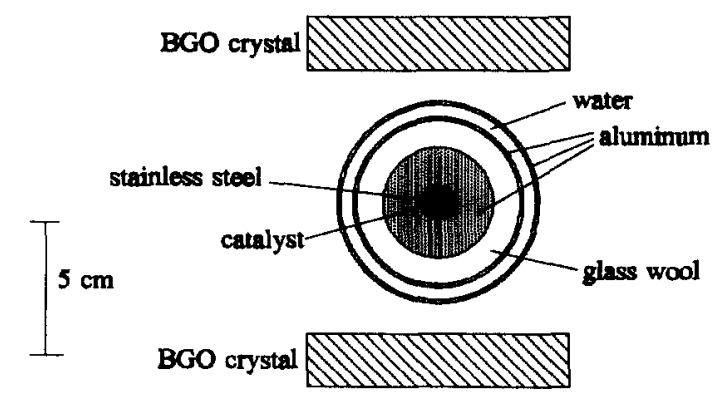

Fig. 1 Cross-section of the geometry used in the simulations

Scintillators involved in the simulations include $\mathrm{NaI}, \mathrm{BaF}_{2}$ and $\mathrm{BGO}\left(\mathrm{Bi}_{4} \mathrm{Ge}_{3} \mathrm{O}_{12}\right)$; the FWHM energy resolution for annihilation photons ( $511 \mathrm{keV}$ energy) is assumed to be 8,13 and $25 \%$ respectively. The detector consists of two banks of each 10 scintillation crystals; the crystals lie next to each other. The annihilation positon of a positron is reconstructed by the intersection of the line between the two crystals which detected a photon above the energy treshold (respectively 450, 430 and $380 \mathrm{keV}$ for $\mathrm{NaI}, \mathrm{BaF}_{2}$ and $\mathrm{BGO}$ ) and the catalyst tube. Reconsturctions between only opposite crystals, all crystals or combinations of crystals can be taken into account. The detector performance is tested by reconstructing the position of a point source in the catalyst which emits positrons with zero kinetic energy.

The results showed that BGO offers a significantly higher sensitivity than the other two scintillators, at the expense of a somewhat worse ratio between proper and misjudged reconstructions (taking into account reconstructions between a crystal and the opposite crystal and the one adjacent to the opposite). Therefore BGO has been chosen as a scintillator for the detector.

With BGO as scintillator the influence of the crystal size on the detector performance was simulated. For a crystal width of $5 \mathrm{~mm}$ (the dimension in the direction of the axial reactor flow) the influence of the crystal thickness (the dimension in the direction of the two crystal banks) and the crystal length on the sensitivity and ratio of proper and misjudged reconstructions was studied. Fig. 2 shows an (expected) rise of the detector sensitivity with crystal thickness.

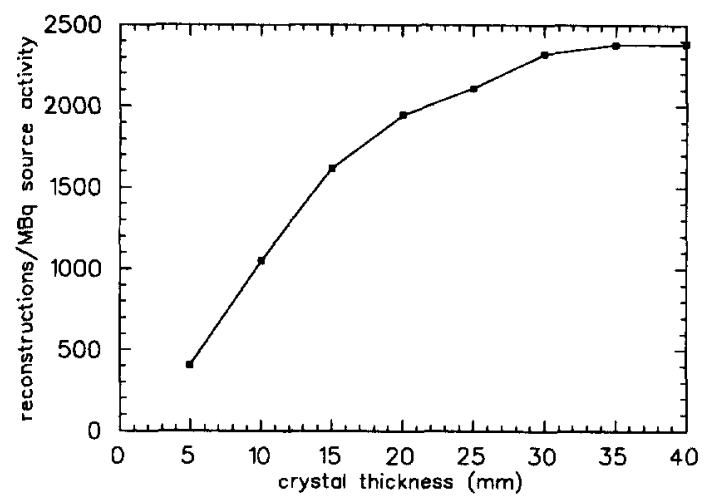

Fig. 2 Detector sensitivity as a function of crystal thickness

Increasing the length of the crystals worsens the ratio between proper and misjudged reconstructions (Fig. 3).

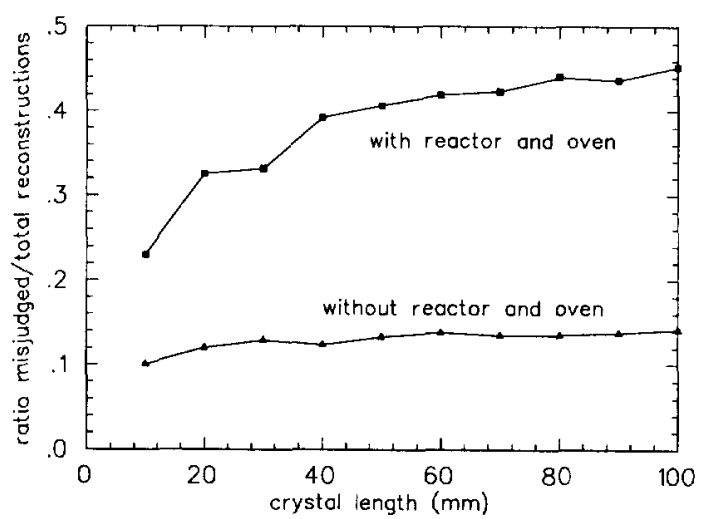

Fig. 3 Ratio between misjudged and total number of reconstructions as a function of crystal length

This is caused by Compton scattering in the reactor and oven: with long crystals the solid angle is so large that a photon scattered under a small angle (and thus with an energy higher than the threshold energy) has a fair chance to be still detected in coincidence with the other photon created upon 
annihilation. In spite of this degradation of detector performance the choice was made for $100 \mathrm{~mm}$ long crystals to achieve maximum sensitivity $(100 \mathrm{~mm}$ is assumed to be the maximum length to detect sufficient light from the BGO-crystal); the two detector banks can be moved away from each other to decrease the effect (at the cost of the detector sensitivity).

The flexibility to be able to measure the activity distribution in catalyst reactors of different types and sizes is obtained by the use of individual detection elements, each with a BGOcrystal. The 20 detection elements ( 2 banks of 10 ) can be individually positioned and fixed in a detector frame to adjust the detector configuration to different reactor lengths and to permit the installation of collimators. To preserve the possibility for close packing of the crystals needed for the position resolution of a few $\mathrm{mm}$ and used in the simulations, a special slit shape type photomultiplier (Hamamatsu R2937) has been chosen. The photomultiplier width of $7.5 \mathrm{~mm}$ allows for close packing of the detection elements by positioning the photomultipliers alternately on one of the two small crystal faces, without using a lightguide. The $20 \times 5 \mathrm{~mm}^{2}$ photocathode of the photomultiplier restricts the crystal thickness to $20 \mathrm{~mm}$, but this is enough to absorb most of the annihilation photons (Fig. 2).

For optimal light collection all crystal faces are polished, except for the face opposite to the photomultiplier which is made rough. The crystals are wrapped in teflon foil and aluminized mylar foil for improved light collection, and in black PVC-tape for sealing from light from the surroundings. The photomultiplier with its resistor network for the distribution of the high voltage over the dynodes is mounted in an aluminum housing; the scintillation crystal is connected to the photomultiplier via an opening in the housing and is kept in position by a supporting arm (Fig. 4). Optical coupling is achieved with silicone grease. The wrapped crystals are about $6 \mathrm{~mm}$ wide, the housing is $10.5 \mathrm{~mm}$ wide, so the alternate positioning of the housing at the left and right side of the crystals allows the crystals to lie next to each other.

The dimensions of the BGO-crystals and of the whole detector are summarized in Table 1.

Table 1 Detector dimensions

\begin{tabular}{|l|c|}
\hline BGO-crystal width & $5 \mathrm{~mm}$ \\
\hline BGO-crystal thickness & $20 \mathrm{~mm}$ \\
\hline BGO-crystal length & $100 \mathrm{~mm}$ \\
\hline Minimum detector length & $62 \mathrm{~mm}$ \\
\hline Maximum detector length & $500 \mathrm{~mm}$ \\
\hline Minimum distance between banks & $70 \mathrm{~mm}$ \\
\hline Maximum distance between banks & $635 \mathrm{~mm}$ \\
\hline
\end{tabular}

\section{ELECTRONICS AND DATA-ACQUISITION}

The electronics system has been built with commercially available units (NIM-standard), except for the preamplifiers which were home-built to be able to optimize the photomultiplier signals. The anode signal from each photomultiplier is fed with a one meter coaxial cable into the preamplifier, which generates for each detected event two signals: an energy signal with height proportional to the integrated charge from the photomultiplier and a filtered timing signal. For the timing signal a shaping time of $155 \mathrm{~ns}$ is used, which seems to be the best compromise between signal risetime (about $40 \mathrm{~ns}$ ), signal height $(80 \mathrm{mV})$ and signal shape (suppression of signal fluctuations caused by the scintillation photons statistics). The timing signals are used for the fast part of the electronic circuit which determines coincidences between the two detector banks. For this level discriminators (Phillips 705) and logic units (Phillips 755) are used: the discriminator pulses from the detection elements of one detector bank are accumulated via logic OR's and tested on coincidence with the other detector bank via logic AND (Fig. 4). The energy signals are amplified and shaped (shaping time $0.5 \mu \mathrm{s}$ ) in a main amplifier and selected on energy by a Single-Channel Analyzer (combined unit, Ortec 590A).

The SCA-pulses (500 ns long) are fed into a home-built 20 channel gated buffer (bit-box) which is gated by a coincidence trigger from the timing circuit (Fig. 4). At each trigger the 20 SCA-connections are read out and their values written into a bitword. If there is a SCA-pulse at a connection at the time of a trigger, a " 1 " is written into the bitword, otherwise a " 0 " is written. A usable event thus has only two " 1 "'s in the bitword; in all other cases the position of the positron annihilation can not be reconstructed.

As the timing circuit is much faster than the energy circuit, the coincidence trigger has to be delayed for about $2.5 \mu \mathrm{s}$ with an active delay line. Synchronization between the 20 detection elements is achieved by equalizing the photomultiplier signal heights (with separate tuning of the the high voltage for each photomultiplier).

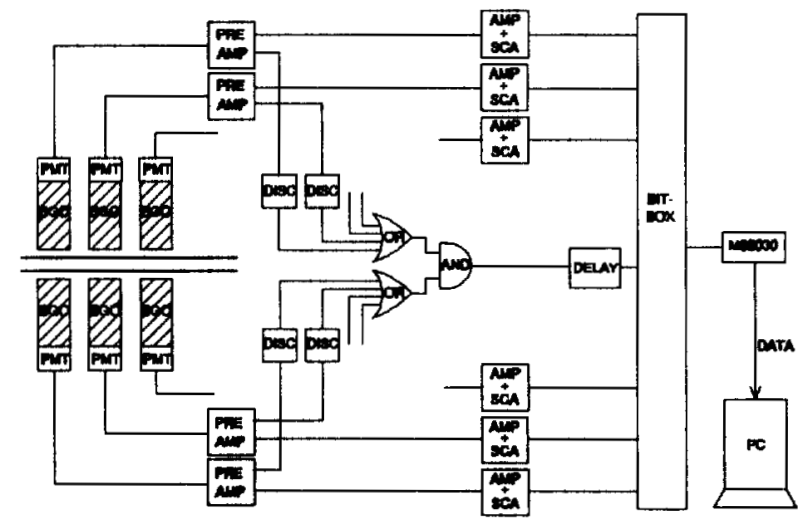

Fig. 4 Scheme of the detector electronics (PMT: photomultiplier tube, AMP: amplifier, DISC: discriminator, SCA: SingleChannel Analyzer) 
For the data-acquisition the standard VME-based computer system of the Physics Department of the Eindhoven University of Technology [5] is used. The system runs with a M68030 processor and is controlled via a PC with an emulator program. The bitwords from the bit-box are alternately stored in one of two local memories. While one memory is collecting data from the bit-box, the collected data in the other memory are copied to a VME-memory, whereafter the data are sorted by event, the activity distribution is on-line reconstructed and monitored on a graphic display and the sorted data are stored. In this way the dead time of the data-acquisition is minimized, and there is almost no loss of measured data. The switching rate between the two local memories can be chosen with the software and determines the detector sampling time; the data processing of one of the two local memories has to take place within this sampling time to prevent loss of measured data. At the moment it is limited to a minimum of 1 second due to the time required for data copying and sorting; the maximum count rate of the system with 20 detection elements is 34,000 events/s.

Stored data contain the number of events per combination of detection elements during each sampling time. After the experiment they are written onto a PC-disk for new and further processing of the data, which allows for off-line monitoring with reconstructions from different detection element combinations.

\section{DETECTOR PERFORMANCE}

The energy resolution of the detection elements for annihilation photons was found to lie between 24 and $30 \%$ FWHM. This is slightly worse than expected. Monte-Carlo simulation of the light transport in the crystal and photomultiplier showed that this is caused by the non-uniform sensitivity of the photomultiplier photocathode, which results in a reduction of the number of photoelectrons coming off the photocathode for light induced near the photocathode edges.

The timing resolution for two individual detection elements is $20 \mathrm{~ns}$ FWHM on average. It can be somewhat improved by lowering the shaping time for the timing signal or by lowering the discriminator treshold level (which is set at $40 \mathrm{mV}$ ), but both measures cause too many fluctuations in the signal and increase the chance for multiple discriminator pulses per detected photon. The coincidence resolving time is determined by the logic OR output pulse width which is $\mathbf{5 0} \mathrm{ns}$. With this pulse width more than $90 \%$ of the coincidences are detected.

The sensitivity and position resolution were measured with a calibrated ${ }^{22} \mathrm{Na}$ source of $1 \mathrm{~mm}$ diameter. The source was placed between the two detector banks in a solid aluminum cylinder of $25 \mathrm{~mm}$ diameter and moved in axial direction. The detector configuration was with the closest crystal packing and the distance between the detector banks was $72 \mathrm{~mm}$. Only events with an energy between 350 and $700 \mathrm{keV}$ were selected for reconstruction. The measurement was also simulated with the EGS4-code to compare the measured and simulated detector performance. Fig. 5 shows the sensitivity and position resolution for two opposite detection elements.

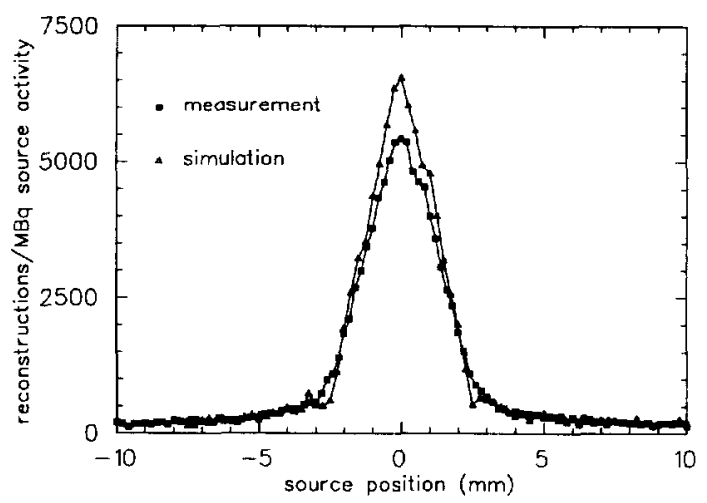

Fig. 5 Measured and simulated sensitivity for a point source in a solid aluminum cylinder as a function of source position

The measured sensitivity is about $15 \%$ lower than the simulated sensitivity, which is probably caused by the fact that not all coincidences are detected in the measurements and by differences in the energy selection settings. The FWHM position resolution is $3.0 \mathrm{~mm}$ in the simulations and $3.2 \mathrm{~mm}$ in the measurements. This may be caused by the fact that in the simulations a point source emitting positrons with zero kinetic energy is used (the $1 \mathrm{~mm}$ diameter ${ }^{22} \mathrm{Na}$ source emits positrons with a maximum kinetic energy of $0.55 \mathrm{MeV}$ ).

The maximum allowable count rate of the detection system has not yet been tested, but will mainly be limited by the coincidence resolving time of 50 ns (giving accidental coincidences at high count rates) and the maximum count rate of the data-acquisition system.

\section{CONCLUSIONS}

A flexible detector system has been designed (with the aid of Monte-Carlo simulations) and built. The detector will be able to measure activity profiles as a function of time in catalyst reactors of a few to $50 \mathrm{~cm}$ 's length. The measured sensitivity and position resolution are close to the ones obtained with the simulations.

Support by NWO (Dutch Organisation for Scientific Research) as part of a Stimulans project is gratefully acknowledged.

\section{REFERENCES}

[1] G. Jonkers, K.A. Vonkeman, S.W.A. van der Wal and R.A. van Santen, Nature, vol. 355, pp. 63-66, 1992.

[2] K.A. Vonkeman, Exhaust Catalysis Studies Using In-situ Positron Emission, Eindhoven University of Technology, thesis, 1990.

[3] M.R. Hawkesworth et al., Nuclear Instruments and Methods, Vol. A310, pp. 423-434, 1988.

[4] W.R. Nelson, H. Hirayama, D.W.O. Rogers, The EGS4 code system, Stanford Linear Accelerator Centre report 265, 1985.

[5] J.H. Voskamp, F.C. van Nijmweegen and A.J. van der Wal, Nederlands Tijdschrift voor Natuurkunde, Vol. A55, pp. 50-55, 1989 (in Dutch). 